\title{
Revisión crítica del consumo en el siglo XXI. ¿Nuevas formas de politizar la ciudadanía?
}

Critical review of consumption in the 21st century. New ways to politicize citizenship?

Loreto Sáenz-de-Ugarte Sevilla • loreto.saenzdeugarte@ehu.eus

DEPARTAMENTO DE SOCIOLOGÍA Y TRABAJO SOCIAL,

UNIVERSIDAD DEL PAÍS VASCO/EUSKAL HERRIKO UNIBERTSITATEA

Recibido: $27 / 12 / 2018$

Aceptado: 04/06/2019

\section{Resumen}

Los últimos estudios sobre las formas de consumo en el siglo xxi identifican un cambio en los comportamientos de compra de la ciudadanía. En concreto, la mayor parte de las investigaciones al respecto coinciden en señalar la existencia de una nueva cultura de consumo y de acción consumidora concebida como práctica política propia de una ciudadanía activa, crítica con el consumismo y orientada por un consumo responsable y sostenible. Este artículo revisa críticamente ese nuevo modelo de consumo que sugiere una transformación en el estatus de la ciudadanía-consumidora, para determinar si el comportamiento de consumo en el siglo xxi es o no alternativo al consumismo, es decir, capaz de emanciparse de la lógica consumista característica de las sociedades económicamente globalizadas.

Palabras clave: Cultura de consumo; ciudadanía-consumidora; consumo político; ciudadanía sostenible.

\section{Abstract}

The latest studies on the forms of consumption in the 21st century identify a change in citizens' purchasing behavior. In particular, most of the studies coincide in the existence of a new culture of consumption and consumer action conceived as a political practice of an active citizenship, critical to consumerism and guided for responsible and sustainable consumption. This paper critically reviews this new consumption model that suggets a transformation in the consumer-citizenship status, to determine whether or not the consumption behavior in the 21st century is innovative and alternative to consumption, that is, capable of emancipating itself from the consumerist logic characteristic of economically globalized societies.

Keywords: Consumption culture; consumer-citizenship; political consumption; sustainable citizenship. 


\section{INTRODUCCIÓN}

A partir de las últimas décadas del siglo xx, se ha registrado y conceptualizado una nueva cultura de consumo asociada a prácticas ciudadanas reflexivas y emprendedoras (Rochefort, 1996 y 1997) que han sido denominadas de diferentes maneras, como consumo consciente (Webster, 1975) у consumo político (Micheletti, 2003; Micheletti et al., 2017). Dicha cultura de consumo orientada fundamentalmente por criterios políticos, éticos, sociales y/o medioambientales parece haberse consolidado en el siglo xxi (Ballesteros, 2010; CIS 2002 y 2010; Alonso, Fernández Rodríguez e Ibáñez Rojo 2013 y 2016). A su vez, se trata de una cultura de consumo asociada a conceptos como ciudadanía activa y ciudadanía crítica.

En ese contexto, este artículo parte de una pregunta fundamental: esa nueva cultura de consumo que sugiere un cambio en el perfil de sujeto consumidor, ¿puede ser una vía para repolitizar la ciudadanía del siglo xxi en un sentido emancipador? A partir de esta pregunta, el objetivo del artículo consiste en esclarecer si el comportamiento de consumo político, ético y responsable social y/o medioambientalmente que varios estudios identifican como consolidado en el siglo xxi es o no innovador y alternativo a la lógica del consumismo.

El artículo se estructura en cuatro partes. La primera parte delimita la noción de cultura de consumo y sus dimensiones; la segunda analiza la principal línea argumentativa de las investigaciones que sugieren que la actividad consumidora es un factor de politización de la ciudadanía en el siglo xxi; la tercera parte se centra en la discusión de la cultura de consumo como factor politizador o despolitizador del ejercicio de la ciudadanía, a partir de la conceptualización propia de la ciudadanía consumidora, en la cual distinguiremos dos tipos: el perfil convencional y el perfil postconvencional. La cuarta y última parte presenta las conclusiones, encaminadas a contribuir a un análisis integral de la cultura de consumo en el siglo xxI.

\section{DELIMITACIÓN DE LA CULTURA DE CONSUMO Y SUS DIMENSIONES}

Como práctica universal, el consumo es inherente a la colectividad humana, de manera que su periodización se remontaría al inicio de las primeras sociedades (Crompton, 1994). Desde una interpretación más restringida del concepto, se entiende que la construcción social del significado y ejercicio cotidiano del consumo adopta distintas formas según los paradigmas de sociedad que se van configurando a lo largo de la historia. Unido a ello, si bien existen varias formas de consumo que pueden convivir en un mismo espacio y tiempo, cada contexto socio-histórico se caracteriza por un patrón de consumo en particular que sobresale respecto a los demás como referencia definitoria de esa sociedad (Slater, 1997). En este sentido, algunas prácticas de consumo significativas son, por ejemplo: 
las formas de autoabastecimiento no mercantilizadas (generalmente en sociedades premodernas); el consumo polarizado, donde el consumo opulento exclusivo de una minoría convive con el subconsumo de una mayoría (en las sociedades occidentales hasta la generalización de políticas keynesianas); la provisión colectiva por encima del consumo privado de mercancías (propia de las sociedades de bienestar); la socialización completa de los consumos (sociedades comunistas); la lógica particular del acto de regalar frente al intercambio de mercancías (economía del don); así como otras formas de consumo de última generación, como los bancos de tiempo y la economía colaborativa.

De todas las modalidades posibles de consumo, el interés de este artículo se centra en el consumo de mercancías, al haberse convertido en la actualidad en la práctica predominante frente a otras formas sociales de provisión, adquisición y utilización de recursos materiales o simbólicos. El consumo mercantilizado constituye una institución social con una influencia sin precedentes en la historia, hasta tal punto de que es el paradigma definitorio del ordenamiento social de las sociedades occidentales a partir de la segunda mitad del siglo xx hasta la actualidad.

La evidencia más destacada de la consolidación de este modelo se encuentra en la propia denominación de sociedad de consumo, atribuida por las diferentes corrientes teóricas tanto a la etapa inicial de ese periodo, como es la etapa fordista y su modelo de sociedad de consumo de masas, como al periodo de la modernidad tardía correspondiente a la etapa post-fordista que, tras la crisis de los años setenta, deviene en el actual modelo de sociedad de consumo segmentado o sociedad de consumidores. Por tanto, la definición de la sociedad de consumo y de los sujetos consumidores está ineludiblemente asociada al modelo de consumo mercantilizado como actividad hegemónica frente a otras modalidades de consumo.

\subsection{El consumo como institución social y fenómeno sociocultural de masas}

Es después de la Segunda Guerra Mundial, con la emergencia del llamado Estado de Bienestar (cuyo momento más álgido se sitúa entre los años cincuenta y sesenta del siglo xx), cuando podemos hablar del consumo como fenómeno social, es decir, como consumo de masas. El Estado de Bienestar representó un nuevo modelo político en el que el Estado abandona la idea del laissez faire para intervenir en la cobertura de las necesidades de interés público, como la creación de infraestructuras o la organización de los servicios sociales hasta entonces prácticamente inexistentes. Como consecuencia de lo anterior, el Estado de Bienestar se convirtió en agente principal de la demanda e impulsor del nuevo modelo económico de desarrollo basado en un consumo masivo de bienes. De forma paralela, el Estado construyó un marco legal para garantizar el consenso social, a través de la negociación colectiva entre la clase trabajadora y el capital. Todo ello tendrá una repercusión social inmediata, como es el acceso generalizado de una mayoría de la población a unas mínimas condiciones materiales de vida y la sustitución del conflicto social por un amplio consenso con respecto al sistema (García Pelayo, 1977: 67). 
Con la saturación del mercado, que dependía hasta entonces del gasto estatal, comienza una nueva etapa en el consumo de masas. El sector público empieza a encontrar límites en su capacidad de promoción del consumo y el sistema de producción busca ampliar el mercado dirigiéndose a un nuevo sujeto consumidor: la ciudadanía. En ese contexto, se fomenta una nueva participación de la ciudadanía en la dinámica del modelo de desarrollo industrial y de crecimiento del mercado (Alonso y Conde, 1994). Es la constitución de la sociedad de consumo, caracterizada por el hecho de extenderse a la mayoría de la población una aparente igualdad en el acceso a los bienes, al mismo tiempo que, por primera vez en la historia, se fomenta en amplios sectores de la sociedad el gasto sin límites, incluso por encima del poder adquisitivo de cada individuo. El endeudamiento privado promovido por instituciones políticas y económicas se convierte así en un fenómeno generalizado y endémico en la mayoría de la población desde esa época y hasta la actualidad.

Tras el primer paradigma de la sociedad de consumo a partir de los años cincuenta del siglo xx, la población occidental adquirió una nueva condición que perdura desde su fase de institucionalización con la sociedad de consumo de masas hasta el actual modelo denominado sociedad de consumidores propio de la transición al siglo xxi. Es en ese contexto de confluencia entre el modelo de economía de mercado fundamentado en el consumo de masas, y el modelo de democracia basada en la materialización de la ciudadanía social (con su carácter redistributivo de la riqueza mediante el pacto social), cuando se consolida el doble estatus de la sociedad civil. Esto es lo que entenderemos por estatus de ciudadanía-consumidora (Sáenz-de-Ugarte, 2015).

\subsection{La dimensión económico-política de la cultura de consumo}

En su nuevo papel benefactor e interventor, el Estado de Bienestar fue adquiriendo aquellas competencias y potestades de la acción política que hasta entonces eran el eje fundamental de las relaciones entre la ciudadanía y las instituciones para resolver el conflicto social y avanzar en el reconocimiento de derechos sociales y laborales. Así, la responsabilidad política de la ciudadanía por garantizar y participar en su desarrollo queda progresivamente relegada a un segundo plano y asumida íntegramente por otros agentes institucionales del pacto social. En ese contexto, el Estado cobra protagonismo mediante la promoción de políticas sociales, legitimándose así como el máximo garante de la provisión de recursos materiales para la inmensa mayoría de la población. En las nuevas condiciones históricas de bienestar material y consenso social generalizado, la ciudadanía del keynesianismo, una vez garantizada la provisión de recursos materiales, se vuelca en conseguir una nueva igualdad en otro marco diferente de actuación: el ámbito del mercado, en el que el consumo es legitimado por el sistema productivo como símbolo de calidad de vida. Se construye así uno de los grandes valores que legitiman el Estado de Bienestar: el acceso a un consumo «democrático» como símbolo de igualdad social y, a su vez, de calidad de vida (Galbraith, 1975).

Progresivamente, el ámbito económico tenderá a sustituir al ámbito político en la garantía de igualdad y de los derechos de ciudadanía. Habermas, en su obra Problemas de legitimación 
del capitalismo tardío (1975) explica las consecuencias del consumismo y el avance de la despolitización de la ciudadanía mediante su concepto de privatismo civil y privatización de la vida cotidiana: «Privatismo civil significa que los ciudadanos se interesan por los rendimientos fiscales y de seguridad social del sistema administrativo, y participan poco —aunque de acuerdo con las posibilidades institucionalmente prescritas - en el proceso de legitimación (elevada orientación hacia el output versus escasa orientación hacia el input). El privatismo civil corresponde entonces a las estructuras de "lo público" despolitizado. El privatismo familiar y profesional es complementario del anterior; consisten en la orientación de las familias hacia los intereses del consumo conspicuo y del tiempo libre, por una parte, y por otra hacia la carrera profesional en la competencia por el estatus» (Habermas, 1986 [1975]: 96).

Lo anterior supone que la búsqueda de satisfacción real de los individuos a través de acciones políticas o sociales de carácter comunitario, como son los logros en la obtención y desarrollo de derechos cívico-políticos y sociales, es sustituida por otro tipo de satisfacción aparente y de carácter privado por medio de la adquisición de bienes y servicios. Su adquisición permitiría revestirse de objetos y actividades que simbolicen aquellos valores que «coticen" más alto en la cultura convencional del modelo de sociedad de consumo o sociedad del bienestar. Con ello, la finalidad es participar en un juego simbólico donde se marcan las diferentes posiciones sociales, estableciéndose de esta manera un baremo para medir los diferentes niveles de prestigio y respetabilidad que confiere cada posición respecto del grupo o comunidad de pertenencia y de referencia y, en última instancia, la posibilidad de medir un mayor o menor grado de poder de decisión en la sociedad general.

\subsection{La dimensión socio-cultural del consumo}

La cohorte de edad nacida con el desarrollo del Estado de Bienestar, conocida como la generación del baby-boom, ha sido la primera socializada íntegramente en el modelo de sociedad de consumo de masas. Se trata de una generación que ha ido resolviendo sus opciones vitales y posicionándose — de manera integradora o rupturista - ante la confluencia en esa sociedad de los valores mercantiles y los valores de la ciudadanía democrática y social. Dicha confluencia conforma un sistema normativo dual hegemónico gestionado de una manera contradictoria por las principales instituciones político-económicas.

En el contexto histórico de socialización de esa generación, los avances formales y materiales de la ciudadanía social son percibidos mayoritariamente como sinónimo de la democratización en el acceso al consumo de mercancías. El progreso social y la abundancia material logrados, son proyectados por el pacto institucional entre Estado de Bienestar y el mercado como la alianza aparentemente indisociable entre los valores mercantiles y los valores de la ciudadanía democrática y social. A su vez, la generación baby-boomer se encuentra ante un cambio de los criterios definitorios de estatus social, por el cual cada vez es menos importante el nivel que se ocupa en el sistema productivo, y en su lugar, cobra mayor importancia como criterio de reputación social la distinción en las elecciones de consumo, fundamentalmente de consumo simbólico. 
La transición del interés de la ciudadanía por los asuntos públicos a un interés cada vez mayor por los asuntos de carácter privado se hace, paradójicamente, aún más evidente tras el agotamiento del modelo de crecimiento económico basado en el consumo. La recesión económica de los años setenta y los posteriores momentos de recuperación e involución de los ochenta y los noventa, respectivamente, apuntaban en este sentido. El consumo mercantilizado, que está configurado por valores e instituciones que definen la modernidad occidental (como la elección, el individualismo y las relaciones de mercado), se convierte en la práctica principal que da sentido a la vida cotidiana de la ciudadanía postkeynesiana. Para Bocock, la consecuencia socio-cultural más relevante de la hegemonía del consumo mercantilizado es el consumismo, que define como la «ideología activa que otorga el sentido de la vida a la adquisición de productos y experiencias organizadas [que] impregna el capitalismo moderno. La ideología del consumismo sirve tanto para legitimar el capitalismo como para motivar a los individuos a convertirse en consumidores no solo en la realidad, sino también en la fantasía. Es más, ha provocado el incremento de la alienación» (Bocock, 1993: 77-78).

Más recientemente, la crisis mundial iniciada en 2008 muestra de nuevo la consolidación de un proceso de despolitización de la sociedad civil, la cual prácticamente ha cedido la defensa de sus derechos y el ejercicio de sus responsabilidades a las instituciones. El gran retardo de una respuesta civil generalizada ante los sucesivos recortes de derechos básicos supone la culminación de una ciudadanía acomodada a la cultura de consumo forjada en el keynesianismo.

\section{APROXIMACIÓN A LAS TEORÍAS DEL CONSUMO EN EL SIGLO XXI}

Las principales teorías de consumo del siglo xxi interpretan el concepto de ciudadanía-consumidora de una forma que entiende el consumo como factor de repolitización de la ciudadanía. Conceptos como consumo político, ético y/o ecológico, y austeridad en el consumo, entre otros, forman parte de la corriente principal de las investigaciones, que coinciden en dar protagonismo al activismo en el consumo como una nueva vía que capacitaría a la ciudadanía contemporánea para un ejercicio político emancipador. Una aproximación a las investigaciones sobre la capacidad política de la ciudadanía en su actividad consumidora nos permite identificar dos tipos de enfoques principales.

\subsection{Enfoque centrado en el consumo político}

Las evidencias sobre los cambios en los comportamientos de compra en el siglo xxi en las sociedades modernas registran en los últimos años un incremento de nuevas formas de activismo político mediante el consumo. El consumo político se define como el «uso del mercado como un espacio para la política» que, al igual que otras formas de acción, precisa de tiempo, recursos económicos y competencias cívicas (Verba et al., 1995; Micheletti, 2003; 
Micheletti et al., 2017). La ciudadanía elige a productores y productos y servicios en función de las valoraciones éticas, políticas y medioambientales que hace de los procedimientos utilizados por las empresas y los gobiernos (García Espejo y Novo Vázquez, 2017). En este sentido, el concepto de consumo político está estrechamente ligado al de consumo consciente que ya fue acuñado en la década de 1970, para hacer referencia a «aquellos consumidores que tienen en cuenta las consecuencias públicas de su consumo privado o que tratan de usar el poder de compra para lograr que se produzca un cambio social» (Webster, 1975: 188).

El consumo político se considera una forma de participación política cada vez más importante en la práctica ciudadana de las democracias occidentales, en la cual se desdibujarían los límites entre lo privado y lo público (Dalton, 2008; Strømsnes, 2009). Por tanto, pese a que el consumo es un acto privado, el sujeto consumidor es visto como un activista político (Zamwel et al. [2014: 201], citado en García Espejo y Novo Vázquez, 2017: 61).

De acuerdo a la revisiones bibliográficas sobre el tema (Novo Vázquez, 2014; García Espejo y Novo Vázquez, 2017), encontramos que, desde los primeros estudios hasta la actualidad, el consumo político ha sido fundamentalmente medido en sus dos dimensiones principales: buycott y boicott (Beck et al., 1997; Micheletti, 2003). A partir del artículo de Dietlind Stolle, Marc Hooghe y Michelle Micheletti (2005): Politics in the Supermarket: Politics Consumerism as a Form of Political Participation, el de mayor impacto en este tema, se han sucedido las encuestas centradas en el consumo político y, en particular, en las dimensiones mencionadas. Así, se estima que «entre un $22 \%$ y un $47 \%$ de americanos y europeos respectivamente participan en el consumo político» (Strømsnes, 2009; Baek, 2010; y Copeland, 2013). En el Estado español, según datos del Centro de Investigaciones Sociológicas (CIS 2002 y 2006), se registra un comportamiento vinculado al consumo político que ha tenido una evolución creciente en los últimos años. De acuerdo a ese organismo, en el año 2002 el 12\% de la ciudadanía española había realizado alguna forma de buycott, cifra que en 2010 se duplicó. A su vez, en 2002 un 6\% de la población afirmaba haber participado en acciones de boycott, mientras que en 2010 el porcentaje se elevó al 20\%. A pesar de esta tendencia, las revisiones bibliográficas sobre estas cuestiones anteriormente citadas, reconocen la existencia de investigaciones cualitativas que señalan la dificultad que presentan las opciones de consumo político o ecológico para consolidarse como patrón de comportamiento en la ciudadanía española, como consecuencia fundamentalmente de la crisis económica. En este sentido, destacamos la publicación de Alonso, Fernández Rodríguez e Ibáñez Rojo (2013).

En general, la tendencia de la mayoría de las investigaciones es medir el consumo político desde un punto de vista cuantitativo y a partir de la búsqueda de correlaciones entre el consumo y distintas variables socioeconómicas. Si bien todas ellas aportan un análisis descriptivo exhaustivo sobre el tema, sin embargo no permiten afirmar que el consumo político sea una tendencia capaz de enfrentar o incluso revertir los efectos de la cultura de consumo o consumismo. En este sentido, cada vez se están teniendo más en cuenta los análisis cualitativos en los estudios vinculados a este enfoque, como comienzan a introducir autoras como García y Novo (2017) y Alonso, Fernández Rodríguez e Ibáñez Rojo (2016). 
INGURUAK [66] | 2019 | 20-34

Revisión crítica del consumo en el siglo XXI | Loreto Sáenz-de-Ugarte Sevilla

\subsection{Enfoques centrados en la austeridad en el consumo}

A la par que el aumento en el consumo político, otras investigaciones apuntan a la emergencia de comportamientos asociados a una vuelta a la austeridad en el consumo que, a su vez, algunos estudios ven como una forma de instrumentalizar el consumo a favor de intereses de clase.

Los estudios relacionados con la vuelta a la austeridad en el consumo o a la frugalidad, que han acuñado conceptos como downshifting consumer, strategic poverty y low-income lifestyles (Demetry, Thurk y Fine, 2013; Nelson, Rademacher y Paek, 2007), subrayan el auge de una tendencia de consumo basada en un modo de vida frugal. En el Estado español, al estudiar esta tendencia, Luis Enrique Alonso, Carlos Fernández Rodríguez y Rafael Ibáñez Rojo (2016) destacan que se trata de un tipo de consumo en el que prevalecen los sentimientos de angustia y el miedo, y en el que se acepta de un modo fatalista la pérdida de poder adquisitivo como preludio de una nueva sociedad más desigual que se va consolidando progresivamente en los países con economía de mercado. Si bien estos autores relacionan la frugalidad con una situación externa como la crisis de 2008, que impone restricciones en el consumo, reconocen que también se puede relacionar el consumo frugal con una elección voluntaria o razón estratégica.

Es a nivel internacional donde encontramos una profundización sobre este último aspecto. Algunos estudios señalan que las nuevas formas de consumo forman parte de una estrategia de instrumentalización del consumo a favor de intereses de clase. Argumentan que el consumo basado en un comportamiento partidario de la austeridad tiene más relación con una estrategia a corto plazo que posteriormente permita a quienes lo practican mejorar su estatus, que con una motivación real por la sostenibilidad social y/o medioambiental (Demetry, Thurk y Fine, 2013).

Esta estrategia utilitarista se diferenciaría de la estrategia a largo plazo representada por activistas sociales que ejercen prioritariamente su papel ciudadano en el ámbito político y de manera vicaría en el mercado (Demetry, Thurk y Fine, 2013; Nelson, Rademacher y Paek, 2007). Como veremos, es el comportamiento de consumo de estos sujetos el que más se ajusta a una manera de extender el ejercicio de la ciudadanía a todos los ámbitos de la vida cotidiana, incluido el consumo, y no al revés.

\section{DISCUSIÓN SOBRE DEL CONSUMO DEL SIGLO XXI COMO FACTOR DE POLITIZACIÓN O DESPOLITIZACIÓN DE LA CIUDADANÍA}

En la segunda mitad del siglo xx, y en paralelo al desarrollo de la sociedad del bienestar, los logros obtenidos por la implantación de la ciudadanía social tuvieron como consecuencia no esperada la generalización de la idea de que el bienestar social y la democratización 
del consumo son dos realidades con una misma procedencia: la economía de mercado, quedando en el olvido el factor determinante de ese progreso social, como es la acción social organizada y orientada a la consecución de los intereses generalizables. Como nos recuerda Crompton (1994: 202), no podemos olvidar que solo mediante la lucha y la reivindicación social se han alcanzado tanto la ciudadanía industrial (los derechos a la negociación colectiva) como la ciudadanía social (en particular los derechos al bienestar y a la redistribución de la propiedad privada), al mismo tiempo que ambas son fuente de permanente conflicto con los intereses del orden capitalista dominante. Así, la despolitización ciudadana que conlleva la cultura de consumo supone el olvido de las conquistas sociales de los movimientos sociales organizados y del pacto social entre la patronal y el movimiento obrero mediado por el Estado de Bienestar (Galbraith, 1975 [1958]; Crompton, 1994; Slater, 1997).

Sin embargo, como hemos visto, las principales teorías de consumo del siglo xxi interpretan el concepto de ciudadanía-consumidora de una forma que entiende el consumo como factor de repolitización de la ciudadanía. García Canclini, con la publicación de Consumidores y ciudadanos. Globalización y conflictos multiculturales (1995), ejemplifica esa nueva vía de exploración del consumo vinculado con la evolución del concepto de ciudadanía. Este autor se interesa por cómo los cambios en la manera de consumir han alterado las posibilidades y formas de ejercer la ciudadanía, concluyendo que: «Junto con la descomposición de la política y el descreimiento en sus instituciones, otros modos de participación ganan fuerza. Hombres y mujeres perciben que muchas de las preguntas propias de los ciudadanos - a dónde pertenezco y qué derechos me da, cómo puedo informarme, quién representa mis intereses- se contestan más en el consumo privado de bienes y de los medios masivos que en las reglas abstractas de la democracia o en la participación colectiva en espacios públicos» (García Canclini, 1995: 13). El autor considera que: «vincular el consumo con la ciudadanía requiere ensayar una reubicación del mercado en la sociedad, intentar la reconquista imaginativa de los espacios públicos, del interés por lo público. Así el consumo se mostrará como un lugar de valor cognitivo útil para pensar y actuar significativa, renovadoramente en la vida social» (ibíd.: 55).

Josée Johnston, en su artículo The Citizen-Consumer Hybrid: Ideological Tensions and the Case of Whole Foods Market (2008), cuestiona que el consumo pueda ser un factor de politización de la ciudadanía del siglo xxi. En su estudio, centrado en el mercado de alimentación, concluye que la ciudadanía-consumidora ética y ecológica puede no ser tan coherente con la idea de ciudadanía entendida en su acepción más literal, ya que expresa un tipo de consumo que tiene un fuerte componente de clase social. Es decir, sería un consumo sostenible asociado a nuevas formas de distinción por parte de una clase social con alto capital económico y cultural. La máxima de «votar con tu dólar» quedaría así desmontada, ya que esa práctica está ligada al habitus de consumo de la elite, más que al de aquellos que carecen de un capital económico y cultural que les permita realizar elecciones de compra para sí mismos y sus familias (Johnston, 2008: 258).

En línea con Johnston, plantear el activismo en el consumo como una nueva salida para legitimar el ejercicio de la ciudadanía significa adoptar una perspectiva que, si bien supone 
una aportación para entender las nuevas formas de consumo frente al consumismo clásico, tiene como limitación la ausencia de un análisis integral del significado último del concepto hibrido de ciudadanía-consumidora, en el que lo dos términos que lo componen resultan ser mutuamente incompatibles. Por ello, el papel que atribuyen ese tipo de teorías a la ciudadanía en su acción consumidora presenta una contradicción difícil de resolver.

Por un lado, el significado de ciudadanía se construye y desarrolla fundamentalmente en el ámbito político de «lo público», el cual, a su vez, está orientado por el principio de responsabilidad colectiva ante la consecución de intereses generalizables. Por otro, el significado de consumidor o consumidora está estrechamente ligado al ámbito del mercado en el que lo prioritario es la lógica del beneficio particular. En el contexto actual, el concepto hibrido de ciudadanía-consumidora supone una contradicción, al apelar a un sujeto orientado de manera simultánea por dos lógicas contrarias, y más bien indica la vigencia de la idea habermasiana de privatización de la vida cotidiana o privatismo civil. Consumir, como principal actividad cotidiana (consumismo), implica una desafección política. Por el contrario, el ejercicio de la ciudadanía por encima de la lógica mercantil implica cuestionar y buscar alternativas a una racionalidad meramente instrumental como es la del mercado que, en su expresión más radical, está representado en el actual modelo de globalización económica neoliberal.

En nuestro caso, la conceptualización de la ciudadanía-consumidora que proponemos se basa en la relación indisociable entre la dimensión socio-cultural de la cultura de consumo, vincula a la agencia, y su dimensión económico-política, vinculada a la estructura, y esto debido a que ambas dimensiones forman parte del proceso de socialización de la ciudadanía. Consideramos que la inclusión de la socialización como variable explicativa es relevante y necesaria debido a su estrecha relación con la configuración del comportamiento ciudadano-consumidor (Saenz-de-Ugarte, 2015).

Así pues, identificamos la existencia de dos perfiles de ciudadanía-consumidora. En primer lugar, el perfil de ciudadanía-consumidora convencional, que se define por una mentalidad particularista expresada por un comportamiento ciudadano-consumidor tendente a la privatización de la vida cotidiana y afín al modelo de economía de mercado. A su vez, identificamos la existencia de un perfil de ciudadanía-consumidora postconvencional, que se define por una mentalidad universalista y plural expresada por un comportamiento ciudadano-consumidor afín al paradigma de la ciudadanía plena, en definitiva un comportamiento significado por el compromiso con el logro de intereses generalizables, es decir, activa, crítica y socialmente responsable. En este perfil destaca el alto grado de coherencia en sus planteamientos alternativos al statu quo, y el grado máximo de desarrollo moral en términos de Kohlberg (Saenz-de-Ugarte, 2015).

De acuerdo a esta caracterización de los perfiles de ciudadanía-consumidora, y en particular sobre la base de la definición realizada del perfil postconvencional, sostenemos que un tipo de ciudadanía que realmente suponga una alternativa al consumismo requiere ir mu- 
cho más allá de un comportamiento ético o responsable en el consumo. Es necesario incluir los componentes de una mentalidad y un comportamiento caracterizados por la acción social organizada y orientada a la consecución de los intereses generalizables más allá del ámbito del mercado y en aras de la sostenibilidad medioambiental y social.

El concepto hibrido de ciudadanía-consumidora en el que se apoyan muchas de las investigaciones sobre el consumo de los últimos años, está en realidad más próximo a unas metas de ciudadanía que podrían estar reproduciendo los tres elementos de la ideología consumista: la elección del consumidor/a, la distinción y el cornucopianismo ecologista ${ }^{1}$ (Johnston, 2008: 262). Como resultado, las nuevas formas de consumo pueden llegar a convertirse en nuevas formas de distinción social o, al menos, representar un ejercicio ético y consciente solo apto para quien tiene acceso al consumo ilimitado de mercancías.

\section{CONCLUSIONES: HACIA UN ANÁLISIS INTEGRAL DE LA CULTURA DE CONSUMO EN EL SIGLO XXI}

El fenómeno social del consumo mercantilizado se ha convertido en uno de los indicadores más representativos de la lógica cultural que sustenta la modernidad tardía desde su modelo de bienestar social hasta nuestros días. Por tanto, toda investigación sobre el consumo actual requiere plantear una relación directa con la modalidad de consumo mercantilizado y con los fenómenos asociados a él, como son la sociedad de consumo, el consumismo y la cultura de consumo en tanto factores vinculantes y sinónimos entre sí. A esto añadimos que el consumo tiene un carácter multidimensional, de forma que sería igualmente incompleto cualquier análisis que no estudie el fenómeno de una manera integral, es decir, relacionándolo con las dimensiones principales que vertebran toda realidad social, como son la dimensión económica, política, social, cultural.

La cultura de consumo está estrechamente ligada una doble lógica: la de la modernidad —materializada en los principios de la democracia y la ciudadanía social—, y la de la economía de mercado. Así, a partir de la Segunda Guerra Mundial, la implantación por primera vez en la historia de ambas lógicas de manera conjunta e institucional con el Estado de Bienestar, tuvo como resultado la creación de un nuevo estatus para la ciudadanía en las sociedades occidentales: el estatus de ciudadanía-consumidora. Este punto de partida significa que la cultura de consumo es heredera de una planificación institucional que es al mismo tiempo política y económica. Este hecho cuando menos cuestiona que la cultura de consumo pueda representar,

\footnotetext{
1 Etimológicamente «cornucopia» significa cuerno de la abundancia, ya que en la mitología clásica, cornucopia es el cuerno de la cabra Amalthea que contiene comidas y bebidas sin fin. Así, la imagen que los cornucopianos se quieren atribuir es la de la riqueza o la abundancia continua. Esta autoasignación es una toma de posición frente a los demás grupos ambientalistas — como los ecologistas radicales y los ambientalistas moderados-, que están a favor de un límite al crecimiento económico por reconocer la finitud de los recursos naturales. Para los cornucopianos no hay que limitar el crecimiento pues la riqueza es verde, y solamente la riqueza, y no la pobreza, podría abastecer los medios para preservar la naturaleza (Chang, 1995: 163).
} 
incluso en la etapa postfordista, un paradigma liberador del modelo anterior de consumo de masas acusado de estandarizar individualidades. Lejos de ser una lógica emancipadora de la ciudadanía, la cultura de consumo desde sus inicios hasta el momento actual representa y reproduce las contradicciones inherentes al modelo de sociedad de los países occidentales.

A pesar de la consolidación de la sociedad de bienestar y la materialización de la ciudadanía social como su logro y paradigma representativo, es el otro paradigma principal de la época, la cultura de consumo, el que influye decisivamente en la acción del individuo, convirtiendo a la mayoría ciudadana en sujeto consumidor a medida que su capacidad como sujeto ciudadano va relegándose a un segundo plano, tanto en el ejercicio de sus derechos como de sus responsabilidades civiles, políticas y sociales. Ambos paradigmas definen la condición de la ciudadanía-consumidora, cuyo primer colectivo representativo lo constituye la generación del baby-boom.

Sin embargo, en esa generación se pueden observar dos tipos diferenciados de capacidades relacionadas con la mentalidad y el comportamiento en relación al modelo hegemónico de sociedad de consumo y su lógica consumista: por un lado, la integración o adaptación y, por otro, el cuestionamiento y la transformación de ese paradigma hegemónico. Estos dos perfiles contrapuestos son los que hemos definido como tipos ideales y conceptualizado como ciudadaníaconsumidora convencional y ciudadanía-consumidora post-convencional, respectivamente. Consideramos que el proceso de socialización y el tipo de transmisión de contenidos y prácticas de valores, vivencias y experiencias en los que intervienen los principales entornos sociales socializadores durante la niñez y la juventud, puede explicar el desarrollo de unas capacidades individuales y sociales que marcan en la etapa adulta el posicionamiento divergente o diferenciado de la ciudadanía de una misma comunidad socio-histórica, como la del baby-boom, ante el doble paradigma de la cultura de consumo y la ciudadanía social activa.

Sobre la base de lo anterior, concluimos que solo un perfil de ciudadanía-consumidora postconvencional, caracterizado por una mentalidad universalista y plural y por un comportamiento afín al paradigma de la ciudadanía plena, tiene mayor posibilidad de generar una alternativa consistente a la cultura de consumo del siglo xxI, actuando de una forma activa, crítica y socialmente responsable en todos los ámbitos de su vida cotidiana con la misma orientación coherente respecto a la consecución de intereses generalizables.

Si bien podemos celebrar la existencia de un perfil de ciudadanía alternativo al modelo de consumo mercantilizador a ultranza, es necesario evitar caer en la complacencia y olvidarnos de la capacidad voraz del mercado para asimilar los comportamientos alternativos a instituciones económicas y políticas burocratizadas y orientadas fundamentalmente por una racionalidad meramente mercantil. La mayor capacidad de influencia en el mercado y en lo político por parte de la ciudadanía mediante sus elecciones de compra, no es más que una nueva forma de integrar al individuo en el sistema, mercantilizando sus iniciativas políticas y, al mismo tiempo, despolitizando sus intereses más allá de la esfera de lo privado y de lo sectorial. 


\section{REFERENCIAS BIBLIOGRÁFICAS}

Alonso, Luis Enrique y Fernando Conde (1994). Historia del consumo en España: una aproximación a sus orígenes y primer desarrollo. Madrid: Debate.

Alonso, Luis Enrique, Fernández Rodríguez, Carlos J. y Rafael Ibáñez Rojo (2016). «Entre la austeridad y el malestar: discursos sobre consumo y crisis económica en España». REIS: Revista Española de Investigaciones Sociológicas, 155, 21-36.

Alonso, Luis Enrique, Fernández Rodríguez, Carlos J. y Rafael Ibáñez Rojo (2013). «From Consumerism to Guilt: Economic Crisis and Discourses about Consumption in Spain». Journal of Consumer Culture, 15(1): 66-85.

Ballesteros, Carlos (ed.) (2010). Ciudadanía del consumo: hacia un consumo más responsable. Cáritas España.

Beck, Ulrich et al. [1997] (2001). Modernización reflexiva. Política, tradición y estética en el orden social moderno. Madrid: Alianza Universidad.

Centro de Investigaciones Sociológicas (2010). Estudio 2736. Internet y participación política. Madrid: CIS.

Centro de Investigaciones Sociológicas (2002). Estudio 2450. Ciudadanía, participación y democracia. Madrid: CIS.

Crompton, Rose Mary (1994). Clase y estratificación, una introducción a los debates actuales. Madrid: Tecnos.

Chang, M. Y. (2005). Cornucopianos: los ultra neoliberales. Sustentabilidad, 179-188.

Dalton, Russell (2008). "Citizenship Norms and the Expansion of Political Participation». Political Studies, 56(1), 76-98.

Demetry, Daphne; Thurk, Jessica y Gary Alan Fine (2013). «Strategic poverty: How social and cultural capital shapes low-income life». Journal of Consumer Culture, 15(1), 86-109.

Galbraith, John Kenneth (1975): La sociedad opulenta. Barcelona: Ariel.

García Espejo, Isabel y Amparo Novo Vázquez (2017). «La emergencia del “consumidor consciente”. Un análisis de la participación política a través de las decisiones de compra». REIS: Revista Española de Investigaciones Sociológicas, 158, 59-76. 
INGURUAK [66] | 2019 | 20-34

Revisión crítica del consumo en el siglo XXI | Loreto Sáenz-de-Ugarte Sevilla

García Canclini, Néstor (1995): Consumidores y ciudadanos. Globalización y conflictos multiculturales. México D.F.: Grijalbo.

García Pelayo, Manuel (1977): Las transformaciones del estado contemporáneo. Madrid: Alianza.

Habermas, Jürgen [1975] (1986): Problemas de legitimación en el capitalismo tardío. Buenos Aires: Amorrortu.

Johnston, Josée (2008). «The Citizen-Consumer Hybrid: Ideological Tensions and the Case of Whole Foods Market». Theory and Society, 37, 229-270.

Micheletti, Michele (2003). Political Virtue and Shopping. Individuals, Consumerism, and Collective Action. Nueva York: Palgrave.

Micheletti, Michele, Follesdal, Andreas y Dietlind Stolle (2017), Politics, Products, and Markets. Exploring Political Consumerism Past and Present. Nueva York y Londres: Routledge. 1. a edición de 2004 en Transaction Publishers.

Nelson, Michelle R., Rademacher, Mark A. y Hye-Jin Paek (2007). «Downshifting consumer = upshifting citizen? An examination of a local freecycle community». The Annals of the American Academy of Political and Social Science, 611: 141-156.

Novo Vázquez, Amparo (2014). «“Consumocracia”. El consumo político como forma de participación de la ciudadanía». Política y Sociedad, 51(1), 121-146.

Rochefort, Robert (1997). Le consumeteurs entrepreneurs. Les nouveaux modes de vie. París: Odile Jacob.

Rochefort, Robert (1996): La societé des consommateurs. París: Odile Jacob.

Stolle, Dietlind; Hooghe, Marc y Michele Micheletti (2005). «Politics in the supermarket: Political consumerism as a form of political participation». International Political Science Review, vol. 26, N. ${ }^{\circ}$ 3, 245-269.

Slater, Don (1997). Consumer Culture and Modernity. Cambridge: Polity Press.

Sáenz-de-Ugarte Sevilla, Loreto (2015). Cultura de consumo y ciudadanía activa en la sociedad contemporánea: discursos y prácticas convencionales y post-convencionales en la generación del baby-boom. Tesis doctoral. Universidad del País Vasco/Euskal Herriko Unibertsitatea. 
Revisión crítica del consumo en el siglo XXI | Loreto Sáenz-de-Ugarte Sevilla

Verba, Sidney, Schlozman, Kay Lheman y Henry E. Brady (1995). Voice and equality. Civic voluntarism in American politics. Harvard University Press.

Webster, Frederick (1975). «Determining the Characteristics of the Socially Conscious Consumer». Journal of Consumer Research, 2, 188-196.

Zamwel, Einat, Sasson-Levy Orna y Guy Ben-Porat (2014). «Voluntary Simplifiers as Political Consumers: Individuals Practicing Politics through Reduced Consumption». Journal of Consumer Culture, 14(2), 199-217. 